\title{
Performance Characteristics of the AmpliSeq Cancer Hotspot Panel v2 in combination with the Ion Torrent Next Generation Sequencing Personal Genome Machine
}

\author{
Kimberly S. Butler ${ }^{a,}{ }^{,}$, Megan Y. L. Young ${ }^{a,}{ }^{,}$, Zhihua Li ${ }^{b}$, Rosalie K. Elespuru ${ }^{a}$, Steven C. Wood ${ }^{a,}{ }^{\dagger}$
}

\begin{abstract}
aU.S. Food and Drug Administration; Office of Medical Products and Tobacco; Center for Devices and Radiological Health; Office of Science and Engineering Laboratories; Division of Biology, Chemistry, and Materials Science; 10903 New Hampshire Ave, Silver Spring, MD 20993, United States

${ }^{b}$ U.S. Food and Drug Administration; Office of Medical Products and Tobacco; Center for Drug Evaluation and Research; Office of Translational Sciences; Office of Clinical Pharmacology; Division of Applied Regulatory Science; 10903 New Hampshire Ave, Silver Spring, MD 20993, United States

"First author: these authors contributed equally to the work.

${ }^{\dagger}$ Corresponding author: 301-796-0243, steven.wood@fda.hhs.gov
\end{abstract}

(C) 2015. This manuscript version is made available under the Elsevier user license http://www.elsevier.com/open-access/userlicense/1.0/ 


\begin{abstract}
Next-Generation Sequencing is a rapidly advancing technology that has research and clinical applications. For many cancers, it is important to know the precise mutation(s) present, as specific mutations could indicate or contra-indicate certain treatments as well as be indicative of prognosis. Using the Ion Torrent Personal Genome Machine and the AmpliSeq Cancer Hotspot panel v2, we sequenced two pancreatic cancer cell lines, BxPC-3 and HPAF-II, alone or in mixtures, to determine the error rate, sensitivity, and reproducibility of this system. The system resulted in coverage averaging $2000 x$ across the various amplicons and was able to reliably and reproducibly identify mutations present at a rate of $5 \%$. Identification of mutations present at a lower rate was possible by altering the parameters by which calls were made, but with an increase in erroneous, low-level calls. The panel was able to identify known mutations in these cell lines that are present in the COSMIC database. In addition, other, novel mutations were also identified that may prove clinically useful. The system was assessed for systematic errors such as homopolymer effects, end of amplicon effects and patterns in NO CALL sequence. Overall, the system is adequate at identifying the known, targeted mutations in the panel.
\end{abstract}

Abstract word count: 200

Keywords: next generation sequencing, cancer hotspot panel, Ion Torrent Personal Genome Machine, mutation detection, systematic error analysis, performance analysis 


\section{Introduction}

Next-Generation Sequencing (NGS) is a rapidly advancing, high-throughput parallel sequencing technology. It

3 has the potential to revolutionize disease research, forensics, and clinical medicine. In the research arena, NGS is being

4 used to gain understanding of disease pathogenesis and to identify new targets for therapy development in a wide

5 variety of cancers and other diseases. For example, the AmpliSeq Cancer Panels are currently being used to identify

6 mutations in Pulmonary Langerhans Cell Histiocytosis as a pathway to understanding the disease and to identify

7 potential treatments (Yousem et al. 2013) and in colorectal cancer patients to identify mutations of clinical relevance

8 (Malapelle et al. 2014). NGS technology is also being evaluated as a potential method to examine mitochondrial DNA as

9 a potential forensic tool (Parson et al. 2013).

Clinically, this technology is being used as a rapid method to identify potential therapies. One area of particular

interest is the use of NGS technology to identify mutations present in the tumors of patients who do not have the most common mutations in their disease and therefore may not respond to the standard treatment. One example of this is the use of NGS in myeloproliferative neoplasms (Tenedini et al. 2014), where the technology is used to identify new treatment targets. Another area of likely use for NGS is in refining patient prognosis. NGS is currently being examined for refining patient prognosis in myeloproliferative neoplasms, gastric cancer and Non-small cell lung cancer (NSCLC) (Fassan et al. 2014; Geurts-Giele et al. 2013; Tenedini et al. 2014). NSCLC can be monoclonal and multifocal or present with multiple primary tumors. The prognosis is different, depending on which type it is, and prognosis can influence treatment options (Geurts-Giele et al. 2013). In hematopoietic cancers, such as Acute Myeloid Leukemia, mutational screening of several genes is part of routine testing and is involved in determining treatment and prognosis. Current testing methods often require examination of several genes individually, which has a high input DNA requirement and high time and resource demands. NGS technology has the potential to streamline the mutation analysis process, reduce the DNA requirement and provide the ability to identify low frequency genetic mutations, as in the investigation of minimal residual disease (Luthra et al. 2014). In addition to treatment and prognosis, clinical research in pancreatic cancer is currently investigating NGS as a method to determine the tumor cellularity versus stromal cellularity, which is a necessary measure for pathological analysis of tumor samples (Song et al. 2012). 
As the use of NGS technology continues to grow, there is also an increasing need to understand the strengths,

weaknesses and overall performance of the technology and of the different available systems. As an investigation into the behavior of an NGS system, we used the Ion Torrent Personal Genome Machine (PGM) System with the AmpliSeq Cancer Hotspot panel v2 to examine the mutations present in two pancreatic cancer cell lines. Pancreatic cancer was chosen because the majority of these cell lines contain multiple mutations in genes present on the AmpliSeq Cancer Hotspot panel v2, in particular KRAS, TP53, CDKN2A and SMAD4 (Deer et al. 2010). KRAS is mutated in the majority of pancreatic cancer cell lines but it is wild type in the cell line BxPC-3. BxPC-3 also presents a unique case for study as it has large scale homozygous deletions in both CDKN2A and SMAD4 which will allow assessment of the performance of the AmpliSeq Cancer panel v2 and Ion Torrent NGS technology in the case of large deletions of genetic material. The other cell line chosen was HPAF-II as it contains both simple mutations as well as small deletions (Table 1). Mixtures of DNA extracted from these cell lines provide a useful model to examine the overall performance and limits of detection of the AmpliSeq Cancer Hotspot panel v2 used with the Ion Torrent NGS system.

\section{Methods}

2.1 Cell lines and cell culture

New aliquots of BxPC-3 and HPAF-II were purchased from American Type Culture Collection (ATCC, Manassas, VA; BxPC3 [CRL-1687], HPAF-II [CRL-1997]) and maintained in culture as recommended by ATCC. RPMI-1640, Eagle's Minimum Essential Medium, fetal bovine serum, DMSO, Phosphate Buffered Saline, Dulbecco's Phosphate Buffered Saline and Trypsin-EDTA solution were purchased from ATCC. Corning 96 well plates, Corning ${ }^{\circledR}$ cell culture flasks (untreated, 25 $\mathrm{cm}^{2}$, vented cap) and NUNC ${ }^{\circ}$ CryoTubes $^{\mathrm{TM}}$ (1 mL, internal thread, polypropylene) were purchased from Fisher Scientific (Hampton, $\mathrm{NH}$ ).

\subsection{DNA isolation and DNA mix preparation}

DNA was isolated from freshly collected cells using the DNeasy Blood \& Tissue Kit from Qiagen (Valencia, CA). DNA concentration was determined using the Broad range Qubit DNA kit and Qubit 2.0 Fluorometer (Life Technologies, Grand Island, NY). After determining DNA concentration, mixed samples of BxPC-3 and HPAF-II DNA were prepared. Mixtures of DNA were prepared at 90:10, 95:5, 99:1 and 99.1:0.1 ratios of BxPC-3 to HPAF-II DNA. HPAF-II and BxPC-3 DNA was also analyzed unmixed to determine the mutation characteristics of the cell lines. 
53 AmpliSeq libraries were prepared as described in MAN0006735 Revision 5.0 (this and subsequent Life Technology 54 protocols are available at www.lifetechnologies.com) using the Ion AmpliSeq Library Kit 2.0 and the Ion AmpliSeq Cancer 55 Hotspot Panel v2 (Life Technologies). Included in this panel were primers for the amplification of regions of the 56 following fifty genes: ABL1, AKT1, ALK, APC, ATM, BRAF, CDH1, CDKN2A, CSF1R, CTNNB1, EGFR, ERBB2, ERBB4, EZH2, FBXW7, FGFR1, FGFR2, FGFR3, FLT3, GNA11, GNAS, GNAQ, HNF1A, HRAS, IDH1, JAK2, JAK3, IDH2, KDR, KIT, KRAS, MET, MLH1, MPL, NOTCH1, NPM1, NRAS, PDGFRA, PIK3CA, PTEN, PTPN11, RB1, RET, SMAD4, SMARCB1, SMO, SRC, STK11,

59 TP53 and VHL. 10ng of each DNA sample was used as the template to prepare the library. Amplified libraries were 60 quantified using the Qubit 2.0 Fluorometer and the High Sensitivity Qubit Assay Kit (Life Technologies). Amplified 61 libraries were assessed for quality (size and concentration) using the Agilent 2100 Bioanalyzer Instrument (Agilent 62 Technologies, Santa Clara, CA) following the Bioanalyzer standard protocol.

$63 \quad 2.3$ Emulsion PCR

64 The AmpliSeq libraries were clonally amplified on to lon Sphere Particles (ISPs) using emulsion PCR following standard 65 Ion Torrent protocols. ISP preparation was performed using the automated Ion Torrent OneTouch2 system following the manufacturer's protocol (MAN0007220 Revision 4.0). The Quit Fluorometer was used to asses ISP quality after ISP preparation but before ISP enrichment. Enrichment of ISPs was performed using the lon Torrent OneTouch ES system and kit following the manufacturer's protocols.

\subsection{Sequencing using the Ion Torrent Personal Genome Machine}

Enriched ISPs were loaded onto 316 chips and a single sample was loaded per chip. Ion Torrent sequencing was performed using the Ion PGM Sequencing 200 Kit v2 and following the standard protocol (MAN0007273 Revision 1.0).

\subsection{Data Analysis}

Alignment of the sequences to human genome build 19 reference genome and base calling were performed using the Torrent Suite software. Identification of variants was performed by the Ion Torrent Variant Caller plugin software. Coverage maps were generated using the coverageAnalysis plugin. 
numbers: SRP767414, SRP767507, SRP767509, SRP767511, SRP767512, SRP767513.

2.7 COSMIC database

80 Mutations found in the unmixed BxPC-3 and HPAF-II were compared to validated mutations listed in the Catalogue of

81 Somatic Mutations in Cancer (COSMIC) Cell Lines Project for both cell lines. The COSMIC cell lines project was accessed 82 June 2014.

83 3. Results

$84 \quad 3.1$ HPAF-II and BXPC-3 cell line analysis

The AmpliSeq Cancer Hotspot Panel v2 targets hotspots for mutation in 50 genes. The number of mutations targeted varies by the gene. The number of targeted mutations was found by examining the variant caller frequency data for each cell line. The number of variants specifically examined by the variant caller software is presented in Table

2. In addition to the targeted mutations, all of the sequences produced are compared by the variant caller software to the human genome reference sequence and 'novel' calls are also made. These 'novel' calls are not targeted variants. data: the software did not call or flag the large homozygous deletions present in the CDKN2A or SMAD4 genes present in the BxPC-3 DNA. Examination of the full data tables also did not reveal this issue. The missing data became evident only when the genes were specifically searched for. An additional plugin, coverageAnalysis, can be used that generates visual coverage maps. These coverage maps clearly show the large areas of missing data in BxPC-3 compared to HPAF-II (Figure $1 \mathrm{~A}$ and $\mathrm{B}$ ).

Figure 1. Coverage maps of HPAF-II, BxPC-3 and mixed DNA samples. Note the lack of coverage $\left({ }^{*}\right)$ in $B$, indicating 98 complete deletion of the regions. 
A. HPAF-II

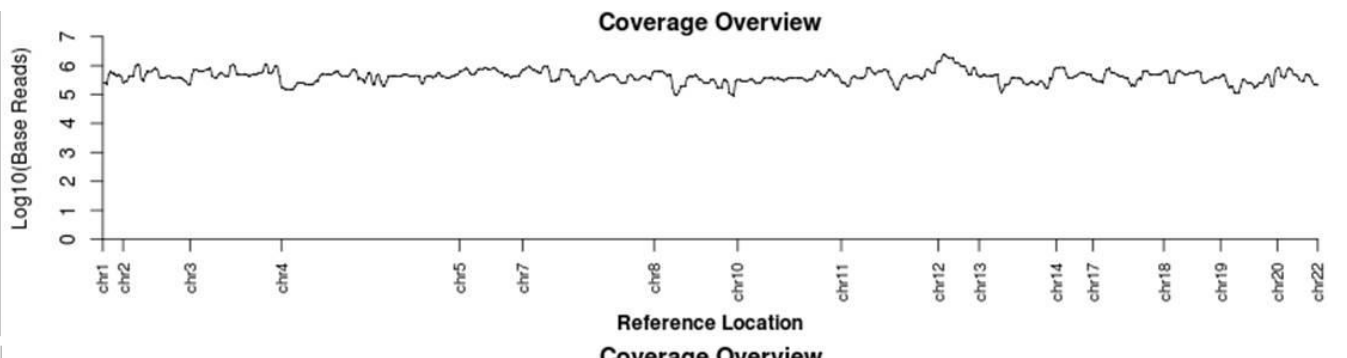

B. BxPc-3

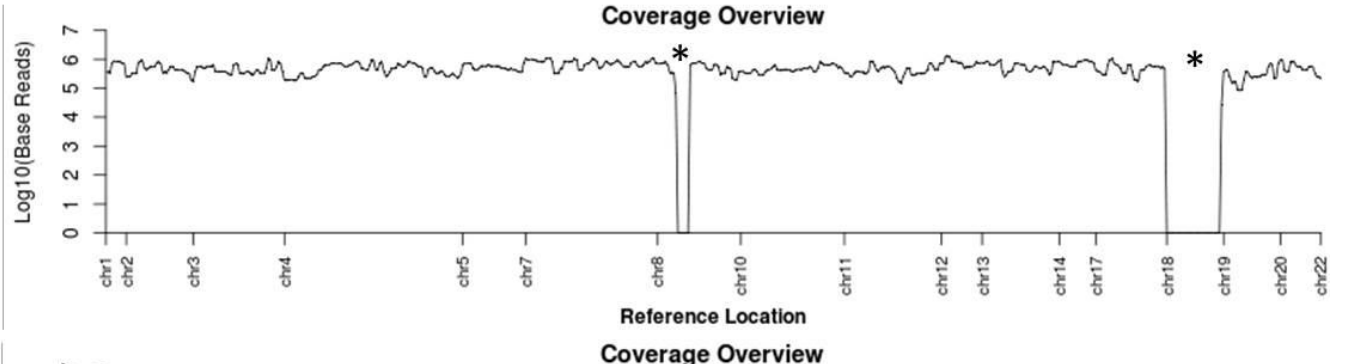

C. $99 \%$ BxPc-3

$1 \%$ HPAF-II

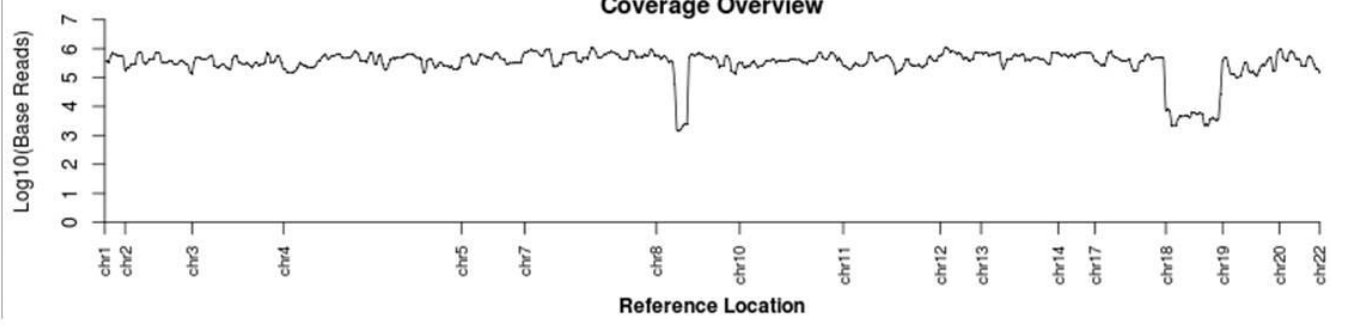

D. $99.9 \%$ BxPc-3

$0.1 \%$ HPAF-II

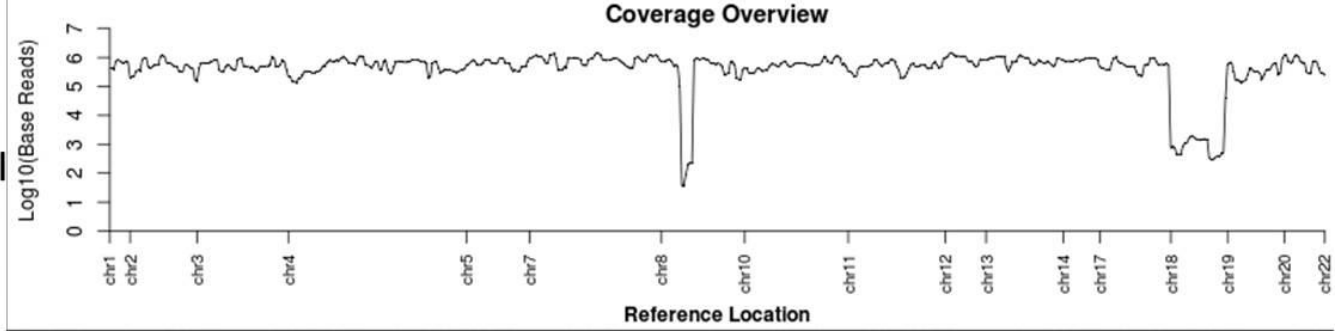

The AmpliSeq variant caller data was compared to the known mutation data from TP53, KRAS, CDKN2A and

SMAD4 for both BxPC-3 and HPAF-II. In BxPC-3, as expected, no mutation was found in KRAS and the expected TP53

mutation was found. However, in addition to the expected mutation in TP53, a second 'novel' mutation was found that has not previously been reported in this cell line. The Ion Torrent coverage map shows the expected homozygous deletion of the SMAD4 and CDKN2A genes in BxPC-3 (Figure 1B). In the HPAF-II cell line no mutations were expected in SMAD4 and none were found. The expected KRAS mutation was found. However, the expected CDKN2A and TP53 mutations were not found. Closer examination of the variant caller data and comparison to the COSMIC allele IDs shows that the mutation in TP53 found in HPAF-II is not a variant that the caller targets as it has never been found in a somatic tumor (COSMIC). Although small deletions in CDKN2A have previously been reported in the literature in HPAF-II, no validated deletion is listed in the COSMIC cell line data base. Although these deletions in CDKN2A were not specified in COSMIC and the TP53 mutation was not specifically looked for by the variant caller, these mutations would show up as 
'novel' mutations because they would be present within the examined regions of the gene. We did not see these 'novel' mutations and there are two possible reasons for this. It is possible that the aliquots of the cell lines we examined, although fresh from the supplier, did not possess the mutations. This seems likely for the CDKN2A deletions, which are not validated. However, a more likely explanation for the TP53 mutation is that the mutation fell outside the regions that were examined. The AmpliSeq Cancer Hotspot panel v2 is a multiplex series of PCR-amplified targets that cover a limited portion of each gene (Table 3). Examining TP53, which is the most highly covered gene, the variant caller looks for 1141 variants, but this corresponds to only $4.85 \%$ of the total gene.

\subsection{Mixed BXPC-3 and HPAF-II DNA sample analysis}

Initial sequencing results from the unmixed BxPC-3 and HPAF-II samples showed an average coverage of approximately $2000 x$ per base. Given this estimate of coverage, it is possible that mutations present as low as $1 \%$ would be visible in the mixed samples. The ability to see mutations in the presence of normal DNA is highly important in actual tumor analysis, as there is often a large normal component to the samples. Mixed DNA samples were created with the ratios of BxPC-3 to HPAF-II of 90:10, 95:5, 99:1 and 99.9:0.1.

There was substantial overlap in the variants called in BxPC-3 and in HPAF-II. Using the standard Torrent Suite variant caller, fifteen variants were called in BxPC-3, of which 5 were unique to BxPC-3. Sixteen variants were called in HPAF-II, of which 7 were unique to HPAF-II (Table 4). In the 90:10 BxPC-3 to HPAF-II sample, all five unique to BxPC-3 variants and all seven unique to HPAF-II variants were called. In the 95:5 BxPC-3 to HPAF-II sample, all five unique to BxPC-3 variants were called, but only six of the seven unique to HPAF-II variants were called. The variant that was not called was only present in HPAF-II at 50.6\%, resulting in a near cutoff level in the 95:5 mixture. In the 99:1 and 99.9:0.01 samples, all five unique to BxPC-3 variants were called while no unique to HPAF-II variants were called.

The depth of coverage for each base in the mixed samples remained the same as the unmixed samples of approximately 2000x. Given this depth of coverage, the HPAF-II mutations should be visible in the 99:1 mixed sample, however these mutations were not found by the Variant Caller using the standard sampling parameters. Some of the sampling parameters were changed to determine whether that would allow detection of any of the unique to HPAF-II variants in the 99:1 BxPC-3 to HPAF-II sample. The first parameter changed was min_allele_freq, which is the minimum allele frequency that must be reached in order to be called as a variant. The default is $0.02(2 \%)$ for SNPs and 0.05 (5\%) 
for INDELs. For both SNPs and INDELs, this was changed to 0.01 , which would represent the theoretical $1 \%$ level. The analysis was performed multiple times with these new parameters, and different results were obtained with each analysis. The results ranged from all seven unique to HPAF-II variants being called to only two unique to HPAF-II variants being called. This likely was due to the parameter downsample_to_coverage. This parameter indicates that the software should only look at the specified number of reads (the default is 2000 and the reads are randomly selected), if the coverage is greater than that value. The downsample_to_coverage parameter artificially and randomly reduces the coverage to increase speed of analysis.

Reanalysis was performed with other parameters changed: gen_min_alt_allele_freq (default 0.035) and gen_min_indel_alt_allele_freq (default 0.1). These two parameters generate a candidate list, to which the min_allele_freq parameter is applied and filters the candidate list to generate NOCALLs. The two gen_...freq parameters were lowered to 0.01 , the $1 \%$ theoretical level, in conjunction with the lowered min_allele_freq parameter. With these parameter settings, only two of the seven unique to HPAF-II variants were called in five reanalyses.

Since $1 \%$ is the theoretical level for the unique to HPAF-II variants if they are homozygous, the parameters were also all lowered to 0.005 , or $0.5 \%$, which is below the theoretical $1 \%$ level for homozygous variants and at the theoretical level for heterozygous variants. With these parameter settings, three of the seven unique to HPAF-II variants were called in five reanalyses. The downsampling parameter was then changed in conjunction with the lowered frequency parameters. The downsample_to_coverage was increased to 4000 and the other parameters were set to 0.05 . The addition of changing the downsampling level did not alter the results obtained. The same three of seven unique to HPAF-II variants were called in five reanalyses with the altered downsampling as were called without altering the downsampling parameter.

\subsection{Reproducibility of data}

In many of the planned uses for NGS technology, particularly those which anticipate high use, the default software will be employed. To assess reproducibility, we examined the 95:5 sample, as it was the most diluted sample that identified the majority of variants using the default software. Three independent replicates of the 95:5 BxPC-3 to HPAF-II mixture were performed. A large pool of the samples at 95:5 was initially mixed, followed by three separate library preparation and sequencing runs. This ratio was chosen because some of the variants seen were heterozygous, 
which would result in a final frequency of about $2.5 \%$, which is near the default cutoff ( $2 \%$ ) for calling variants. All three sequencing runs found the same variants.

One sequencing run found an additional variant not seen in the others. This new variant (chr4: T55972974G) was found at only $5 \%$ and at the same location as another variant (chr4: T55972974A). chr4: T55972974A was present in both BxPC-3 and HPAF-II, but it is homozygous in BxPC-3 and heterozygous (51\%) in HPAF-II. In the two 95:5 replicates without the additional variant at this site, chr4: T55972974A was present at nearly 98\%. In the one 95:5 replicate with the chr4: T55972974G variant, the T55972974A variant was present at 92\%, while the T55972974G variant was present at $5 \%$. When the variant file for the sequencing run with the alternate variant was viewed in Integrative Genomics Viewer (IGV) (Robinson et al. 2011; Thorvaldsdóttir et al. 2012) each G variant was preceded by the insertion of an A. This is likely indicative of a software issue in aligning and reading these sequences.

Besides the reanalyses described above for the 99:1 BxPC-3 to HPAF-II sample, reanalyses with altered parameters were performed for the three 95:5 BxPC-3 to HPAF-II samples. The downsampling parameter was not changed, but the three frequency (..._freq) parameters were changed to 0.05 (unless the default setting was lower, then it was left as default), which is the theoretical $5 \%$ level for a homozygous variant in HPAF-II. This change did not alter the results: the same six of seven unique to HPAF-II variants were called as were called with the default settings. The frequency parameters were then changed to $0.01(1 \%)$, which is below the theoretical $5 \%$ level and should identify the one missing unique to HPAF-II variant (present in the 100\% HPAF-II sample at 50.6\%). With these parameter settings, all of the unique to HPAF-II variants were called.

\subsection{NO CALL analysis, Effects of Homopolymer regions and end of amplicon effects}

In addition to the behavior of the AmpliSeq Cancer Hotspot panel v2 and Ion Torrent on mutation identification in mixed samples, the overall performance of the system is important. In each sample there were some hotspot mutations that were considered NO CALL due to failure to pass an internal quality requirement of the software. We analyzed the NO CALLs for increases in the percentage of NO CALLs by mutation type and by gene. Analysis was done by pooling the data from all samples, however, data from the $0.1 \%$ and $1 \%$ mixed samples on the SMAD4 and CDKN2A genes were excluded as these genes were deleted from the BxPc-3 cell line which provided the majority of the genetic material. In the BxPc-3 cell line alone, no data is present in the variant caller data sheet. The addition of even $0.1 \%$ of 
the HPAF-II leads to limited amplification and recognition by the variant caller that data is present in these two genes, however, almost all hotspots in these genes were NO CALLs, which would inflate the rate of NO CALLs in our analysis. Examination of the NO CALLs by mutation type found the most NO CALLs in the deletion mutation type where $6.9 \%$ of all deletion hotspots targeted by the panel were considered NO CALLs. The next highest rate was with a multinuceleotide polymorphism (2.4\%), followed by single nucleotide polymorphisms (1.1\%), and insertions (0.5\%).

Examination of NO CALL data for the individual genes found the rates to be very low in the majority of genes (Table 5). However, there are several genes in which the rate was quite high. Only five genes had NO CALL rates above $5 \%$. These genes were CDH1 (14.3\%), MPL (12.5\%), NRAS (6.8\%), PDGFRA (9.1\%) and STK11 (5.4\%).

Misidentification of homopolymer regions as insertions or deletions has been seen in the literature with NGS technology and specifically with 454 and Ion Torrent platforms (Bolotin et al. 2012). Additionally, the position of the observed 'mutation' in the amplicon may affect the likelihood that these calls are actually present. To determine if homopolymer regions or positional effects were present in these samples, the novel calls were visually examined in IGV to determine if these calls fell in either a homopolymer region or near the end of a read. Examination of the novel calls showed no positional effects or homopolymer effects.

\section{Discussion}

BxPc-3 and HPAF-II pancreatic cancer cell lines as well as mixed samples of these two cell lines were assessed using the AmpliSeq Cancer Hotspot panel v2 and the Ion Torrent PGM as a vehicle for assessing performance characteristics. The use of cell lines for analyses like these has the advantage of providing large quantities of highquality DNA for repeated analysis and these cell lines have been previously examined using other technologies. Some of the mutations are known, which allows selection of cell lines to assess the performance characteristics in specific situations. Although both cell lines have previously been examined for mutations, the analysis of BxPc-3 and HPAF-II using the AmpliSeq Cancer Hotspot panel v2 provided new data on the mutations present within each cell line. Of these new mutations, some of them were targeted mutations previously identified as important in somatic cancer. For example, BxPc-3 has mutations in targeted genes such as HRAS, KIT, PDGFRA and SMARCB1 that had not been previously identified. In addition to these targeted mutations, likely with clinical implications, other novel mutations were identified that add to the body of knowledge of mutations in these cell lines. Further experimentation using a secondary 
technology will be required to confirm both the targeted and the novel mutations. However, the examination of the cell lines revealed some issues with the use of this technology for studying cell lines and these issues could also apply to the use in human tissue. Although 50 genes are examined, only a limited region of each gene is covered; these regions are selected based on the known important somatic mutations found in human cancers. Thus, mutations expected in the cell lines may not be detected if they fall outside the amplified region and new mutation discovery is limited to those targeted regions. However, the tradeoff is rapid and concurrent detection of mutations in multiple cancer-associated genes in a system that can be multiplexed to conserve materials.

One area of interest was the sensitivity to mutation of the AmpliSeq Cancer Hotspot panel v2 and the lon Torrent PGM system. To assess this, we examined mixed samples of HPAF-II on a background of BxPc-3. Using the standard on board variant caller, we were able to see the presence of a $5 \%$ mutation. A $5 \%$ mutation detection rate in a normal background is consistent with previously reported MiSeq cell line dilution study in AML (Luthra et al. 2014) and with lon Torrent PGM sensitivity using a different AmpliSeq Cancer panel (Singh et al. 2013). However, this is in contrast to the results from two other groups who reported $1 \%$ sensitivity using the Illumina GAllx analysis and $2 \%$ detection using custom primers sets (Hadd et al. 2013) and 1\% AmpliSeq Colon and Lung cancer v1 panel sensitivities (Scarpa et al. 2013). We were able to detect $1 \%$ mutation by modifying the parameters for analysis; however, modification made the data variable from analysis to analysis and would require multiple analyses of each data set. Performing multiple analyses is very time consuming and unlikely to be a viable option in most commercial settings. Overall, our results show that the AmpliSeq Cancer Hotspot panel v2 in combination with the lon Torrent PGM using the basic onboard variant caller is as sensitive as pyrosequencing, such as the Roche 454 platform, which commonly detects a $5 \%$ mutation rate; is more sensitive than Sanger sequencing, which can only detect a 20\% mutation rate (Harrington et al. 2013); and is comparable to other NGS technology. Using the threshold of 5\% mutation, the lon Torrent PGM and the AmpliSeq Cancer Hotspot panel v2 showed reliability in repeated testing of the $5 \%$ mixed cell line sample.

The error rates of the Ion Torrent as well as other NGS technologies have previously been examined. Experimentation using the T-cell receptor of the human immune system demonstrated that the error rate in lon Torrent and 454 was lower than Illumina due to the bridge amplification in Illumina (Bolotin et al. 2012). However, previous use of the Ion Torrent, as well as other sequencing technologies, has found homopolymer regions to be an issue. 
Homopolymer sequencing errors are often identified as deletions or insertions (Seo et al. 2013). As the homopolymer region increases in length, the likelihood of a sequencing error increases (Loman et al. 2012; Quail et al. 2012). Homopolymers are an issue specific to 454 and Ion Torrent platforms, not present in Illumina technology (Bolotin et al. 2012) due to its removable fluorophore. Previous experimentation has shown the rate of homopolymer sequencing error in lon Torrent sequencing was as high as 1.5 indel errors per 100 bases sequenced (Loman et al. 2012). Examination of our data using the lon Torrent in combination with the AmpliSeq Cancer Hotspot panel v2 did not reveal any homopolymer-based errors or any errors caused by mutation position in the amplicon. This may be due to the design of the primers used in the AmpliSeq Cancer Hotspot panel v2 or the location of the mutations. The location of the targeted mutations could fall outside homopolymer regions, thus reducing the possibility of homopolymer sequencing errors. Careful design of primers will reduce the potential for positional effect in amplification for the targeted mutations, although the novel mutations cannot be anticipated.

In addition to the potential for incorrect calls due to homopolymers, there is also the potential to miss a mutation due to sequencing failure. Analysis of the data collected from the BxPc-3, HPAF-II and mixed cell line samples has shown that there is a variable failure rate by gene in the AmpliSeq Cancer Hotspot panel v2. Additionally, there was an increased failure rate in targeted deletion mutations in comparison to the other types of mutations. Previous experimentation on the lon Torrent PGM in the context of standard sequencing has provided specific characterization of the errors in Ion Torrent PGM data (Bragg et al. 2013). This work showed the majority of errors were in insertions or deletions at a rate of $2.84 \%$ (1.38\% after quality clipping) caused by over-calling short homopolymers and under-calling long homopolymers. Using the AmpliSeq Cancer Hotspot panel v2, no homopolymer errors were found, which is likely due to the difference in application as the amplified regions showed very few homopolymers that could contribute to these types of errors. This same group also found a high frequency of insertion and deletion errors, approximately every 1000 bases in reference sequence, which occurred more often with the 314 chips and less often with the 316 chips (Bragg et al. 2013). We did find an increase in inability for the variant caller to determine the presence of deletions, as evidenced by the $6.9 \%$ NO CALL rate in deletion variants. However, we did not see any increase in insertions and had fairly low error rates overall. This may be due to the depth of coverage in using the AmpliSeq Cancer Hotspot panel v2 in comparison to whole exome or genome sequencing. Previous data has found a difference in the necessary depth of 
coverage between insertions or deletions and SNPs. With the lon Torrent PGM, a minimum of 100-300x coverage is required to identify insertions or deletions, while a minimum of 20-30X coverage is required to identify SNPs. The actual required depth for insertions and deletions varies by context, and is higher in the presence of homopolymers (Tarabeux et al. 2014).

Next Generation Sequencing technology provides some advantages over other standard methods of sequence assessment, such as Sanger sequencing or mutant-specific PCR technologies. In particular, the multiplexed capacity allows the ability to identify mutations in genes other than the primary target, which could either affect the response to treatment or suggest an alternative treatment. For example, response to EGFR-based treatment depends on KRAS mutational status so the ability to assess both genes is necessary. As the understanding of cancer continues to expand, more such combinations are likely to be discovered so multiplexed assays provide an economy of scale with regards to both materials and time.

NGS technologies have also been found to be comparable in sensitivity to other methods. For example, Illumina MiSeq technology has a limit of detection comparable to Taqman and high-resolution melting curve analysis and superior detection to Sanger sequencing while still allowing high sample throughput (Nguyen-Dumont et al. 2013). The Ion Torrent AmpliSeq cancer panel has been compared to mutant specific PNA PCR and found to have comparable sensitivity. The AmpliSeq cancer panel had the advantage of covering more sequence than many mutant-specific PCRs and can detect mutations not found by mutation-specific PCR (Kim et al. 2013). Sanger style sequencing is often considered the gold standard in sequence identification; however there is a growing body of evidence that the NGS technologies provide very good concordance in sequence while also having the advantage of multiplexing samples. In mitochondrial DNA sequencing, very good concordance in sequence was found between Sanger-type sequencing and Ion Torrent, only $0.02 \%$ of reads showed a difference and many of these occurred at homopolymer regions (Parson et al. 2013). The concordance is not limited only to standard sequencing; the lon Torrent AmpliSeq cancer panel has been compared to traditional sequencing in several genes and gene regions and good sequence concordance was found (Geurts-Giele et al. 2013). Illumina and Ion Torrent technologies have been directly compared with both tumor and cell line DNA and showed good sequence concordance (Hadd et al. 2013; Luthra et al. 2014). One major difference is that Ion Torrent had a higher rate of detecting SNPs than Illumina, but lon Torrent also had a higher rate of error and more 
false positive calls than Illumina (Quail et al. 2012). The incorrect calling of SNPs is likely coverage-based, with at least 30-40x coverage important for correct SNP calling (Quail et al. 2012), showing the importance of ensuring sufficient coverage for clinical and research samples.

While sequencing technology provides a wealth of information about gene mutations, this technology is not applicable in all situations. One of the most obvious areas for concern is mutations that fall within homopolymer regions of genes. While the standard software may be unable to accurately call mutations in these regions, mutations in homopolymer regions can be accurately detected but require different analysis than the standard used in many academic and clinical labs (Tarabeux et al. 2014). In addition to the concern presented with homopolymer regions, another area of concern is large rearrangements, such as those found in BRCA genes. Similar to homopolymer regions, large rearrangements can be detected using bioinformatics, however, this method requires comparison between samples and a secondary confirmation method in addition to NGS sequencing is recommended (Tarabeux et al. 2014). However, the most important concern is that sequencing is not able to detect expression levels, which is important for identifying gene fusion products such as the YWHAE-NUTM2A/B fusion sometimes found in Endometrial stromal sarcomas. These tumors can show a high level of KIT expression by immunohistochemistry, which is not detectable by Sanger Sequencing of KIT or using the AmpliSeq Cancer Hotspot panel. Increases in expression can be important for prognosis and treatment options (Lee et al. 2014).

The data presented here focused on the Ion Torrent PGM and the AmpliSeq Cancer Hotspot panel v2. However, there are multiple panels available and new panels are being developed. Future work is necessary to determine whether the performance characteristics seen with this panel are shared with other panels. In addition to premade panels, custom panels are being made for diseases like breast cancer and identifying disease susceptibility in blood ${ }^{21}$. Future work should also characterize the performance of custom panels to determine how universal the observed performance characteristics are to a particular NGS technology and to determine how strong the effect of primer design and individual gene region characteristics are to error rate, sensitivity and reproducibility.

Text word count: 5244 


\section{Disclaimer}

The mention of commercial products, their sources, or their use in connection with material reported herein is not to be construed as either an actual or implied endorsement of such products by the Department of Health and Human Services. The findings and conclusions in this article have not been formally disseminated by the U.S. Food and Drug Administration and should not be construed to represent any Agency determination or policy.

\section{Acknowledgements}

The authors thank the Medical Countermeasures Initiative for funding this work. The authors acknowledge E. Mansfield (CDRH/OIR) and P. Potnis (CDRH/ODE) for securing funds from the Critical Path and MCMi Programs to establish the lon Torrent Gene Sequencing Core Laboratory at CDRH/OSEL, where this research was conducted. This study was supported by funds provided by the National Cancer Institute Interagency Oncology Taskforce and the Office of Science and Engineering Laboratories, CDRH, FDA. This project was supported in part by an appointment to the Research Participation Program at the Center for Devices and Radiological Health administered by the Oak Ridge Institute for Science and Education through an interagency agreement between the U.S. Department of Energy and the U.S. Food and Drug Administration.

\section{Author Contributions}

KSB and MYLY performed the experiments and wrote the manuscript. ZL, MYLY, and KSB performed data analysis. SCW and RKE provided guidance and intellectual input throughout the project. KSB and SCW secured funding for this work. All authors reviewed the manuscript.

\section{Competing Financial Interests}

The authors declare no competing financial interests. 
Tables

Table 1. Selected mutations present in BxPC-3 and HPAF-II.

\begin{tabular}{|c|c|c|c|c|}
\hline Cell Line & KRAS & TP53 & CDKN2A & SMAD4 \\
\hline BXPC-3 & WT & 220 Cys & HD & HD \\
\hline HPAF-II & 12 Asp & 151 Ser & $\Delta^{*}$ & WT \\
\hline
\end{tabular}

WT - wild type, HD - homozygous deletion, $\Delta$ - deletion

* deletions in CDKN2A have been reported independently 3 times, however the exact bases deleted were different in each study.

Table 2. The number of mutations targeted in each gene and how many targeted and novel mutations were found in each gene in BxPC-3 and HPAF-II.

\begin{tabular}{|c|c|c|c|c|c|}
\hline \multirow[b]{2}{*}{ Gene } & \multirow[b]{2}{*}{$\begin{array}{c}\text { \# of targeted } \\
\text { mutations }\end{array}$} & \multicolumn{2}{|c|}{ BxPC-3 } & \multicolumn{2}{|c|}{ HPAF-II } \\
\hline & & $\begin{array}{c}\text { targeted } \\
\text { mutations }\end{array}$ & $\begin{array}{c}\text { novel } \\
\text { mutations }\end{array}$ & $\begin{array}{c}\text { targeted } \\
\text { mutations }\end{array}$ & $\begin{array}{c}\text { novel } \\
\text { mutations }\end{array}$ \\
\hline ABL1 & 19 & & & & \\
\hline AKT1 & 6 & & & & \\
\hline ALK & 8 & & & & \\
\hline APC & 135 & 0 & 1 & 0 & 1 \\
\hline ATM & 24 & & & & \\
\hline BRAF & 76 & & & & \\
\hline $\mathrm{CDH} 1$ & 7 & & & & \\
\hline CDKN2A & 107 & & $*$ & & \\
\hline CSF1R & 8 & & & & \\
\hline CTNNB1 & 73 & & & & \\
\hline EGFR & 108 & 0 & 1 & 0 & 1 \\
\hline ERBB2 & 19 & & & & \\
\hline ERBB4 & 13 & & & 0 & 1 \\
\hline $\mathrm{EZH} 2$ & 11 & & & & \\
\hline FBXW7 & 25 & & & & \\
\hline FGFR1 & 2 & & & & \\
\hline FGFR2 & 8 & & & & \\
\hline FGFR3 & 16 & 0 & 1 & 0 & 1 \\
\hline FLT3 & 30 & 0 & 1 & 0 & 1 \\
\hline GNA11 & 5 & & & & \\
\hline GNAS & 12 & & & 0 & 1 \\
\hline GNAQ & 6 & & & & \\
\hline HNF1A & 10 & & & & \\
\hline HRAS & 27 & 1 & 0 & & \\
\hline IDH1 & 12 & & & & \\
\hline IDH2 & 12 & & & & \\
\hline JAK2 & 5 & & & & \\
\hline JAK3 & 6 & & & & \\
\hline KDR & 11 & 0 & 3 & 0 & 2 \\
\hline KIT & 105 & 1 & 0 & & \\
\hline KRAS & 61 & & & 1 & 0 \\
\hline MET & 18 & & & & \\
\hline MLH1 & 1 & & & & \\
\hline MPL & 10 & & & & \\
\hline NOTCH1 & 20 & & & & \\
\hline NPM1 & 28 & & & & \\
\hline NRAS & 35 & & & & \\
\hline PDGFRA & 22 & 1 & 1 & 0 & 1 \\
\hline
\end{tabular}




\begin{tabular}{|c|c|c|c|c|c|}
\hline PIK3CA & 97 & & & 0 & 1 \\
\hline PTEN & 148 & & & & \\
\hline PTPN11 & 28 & & & & \\
\hline RB1 & 18 & & & & \\
\hline RET & 17 & & & 0 & 2 \\
\hline SMAD4 & 31 & & $*$ & & \\
\hline SMARCB1 & 11 & 1 & 0 & 1 & 0 \\
\hline SMO & 5 & & & & \\
\hline SRC & 1 & & & & \\
\hline STK11 & 21 & & & & \\
\hline TP53 & 1141 & 1 & 1 & 0 & 2 \\
\hline VHL & 121 & & & & \\
\hline
\end{tabular}

* Region unflagged by variant calling software, however there is no data present for these regions. These regions are known homozygous deletions.

Table 3. Percentage of each gene covered by targeted amplification of the AmpliSeq panel.

\begin{tabular}{|c|c|c|c|}
\hline Gene & $\begin{array}{c}\text { total length } \\
\text { (bp) }\end{array}$ & $\begin{array}{c}\text { covered } \\
\text { length (bp) }\end{array}$ & $\begin{array}{c}\text { percent } \\
\text { covered }\end{array}$ \\
\hline ABL1 & 173795 & 408 & $0.23 \%$ \\
\hline AKT1 & 26394 & 224 & $0.85 \%$ \\
\hline ALK & 728838 & 230 & $0.03 \%$ \\
\hline APC & 138735 & 826 & $0.60 \%$ \\
\hline ATM & 146268 & 1533 & $1.05 \%$ \\
\hline BRAF & 190752 & 243 & $0.13 \%$ \\
\hline CDH1 & 98250 & 325 & $0.33 \%$ \\
\hline CDKN2A & 26740 & 255 & $0.95 \%$ \\
\hline CSF1R & 60082 & 181 & $0.30 \%$ \\
\hline CTNNB1 & 44445 & 118 & $0.27 \%$ \\
\hline EGFR & 188307 & 892 & $0.47 \%$ \\
\hline ERBB2 & 40523 & 365 & $0.90 \%$ \\
\hline ERBB4 & 1162911 & 843 & $0.07 \%$ \\
\hline EZH2 & 76978 & 85 & $0.11 \%$ \\
\hline FBXW7 & 213984 & 492 & $0.23 \%$ \\
\hline FGFR1 & 57697 & 238 & $0.41 \%$ \\
\hline FGFR2 & 120129 & 440 & $0.37 \%$ \\
\hline FGFR3 & 15561 & 518 & $3.33 \%$ \\
\hline FLT3 & 97319 & 400 & $0.41 \%$ \\
\hline GNA11 & 27047 & 92 & $0.34 \%$ \\
\hline GNAQ & 311029 & 123 & $0.04 \%$ \\
\hline GNAS & 71456 & 218 & $0.31 \%$ \\
\hline HNF1A & 23766 & 177 & $0.74 \%$ \\
\hline HRAS & 3309 & 206 & $6.23 \%$ \\
\hline IDH1 & 18854 & 103 & $0.55 \%$ \\
\hline IDH2 & 18497 & 130 & $0.70 \%$ \\
\hline JAK2 & 143098 & 128 & $0.09 \%$ \\
\hline JAK3 & 23251 & 297 & $1.28 \%$ \\
\hline
\end{tabular}




\begin{tabular}{|c|c|c|c|}
\hline KDR & 47337 & 924 & $1.95 \%$ \\
\hline KIT & 82787 & 854 & $1.03 \%$ \\
\hline KRAS & 45675 & 331 & $0.72 \%$ \\
\hline MET & 125982 & 640 & $0.51 \%$ \\
\hline MLH1 & 72113 & 126 & $0.17 \%$ \\
\hline MPL & 16661 & 118 & $0.71 \%$ \\
\hline NOTCH1 & 51343 & 348 & $0.68 \%$ \\
\hline NPM1 & 23181 & 115 & $0.50 \%$ \\
\hline NRAS & 12431 & 249 & $2.00 \%$ \\
\hline PDGFRA & 920593 & 461 & $0.05 \%$ \\
\hline PIK3CA & 86187 & 1101 & $1.28 \%$ \\
\hline PTEN & 105338 & 843 & $0.80 \%$ \\
\hline PTPN11 & 91182 & 236 & $0.26 \%$ \\
\hline RB1 & 178144 & 992 & $0.56 \%$ \\
\hline RET & 53281 & 611 & $1.15 \%$ \\
\hline SMAD4 & 90228 & 1028 & $1.14 \%$ \\
\hline SMARCB1 & 47556 & 475 & $1.00 \%$ \\
\hline SMO & 24673 & 520 & $2.11 \%$ \\
\hline SRC & 60734 & 103 & $0.17 \%$ \\
\hline STK11 & 22637 & 616 & $2.72 \%$ \\
\hline TP53 & 19149 & 928 & $4.85 \%$ \\
\hline VHL & 12036 & 318 & $2.64 \%$ \\
\hline
\end{tabular}

Table 4. Variant calls in unmixed and mixed HPAF-II and BxPc-3 samples.

\begin{tabular}{|c|c|c|c|c|c|c|}
\hline HPAF-II:BxPC-3 (\%HPAF) & ERBB4 & GNAS & HRAS & KDR & KIT & PDGFRA \\
\hline Undiluted (100\%) & $65.3 \%$ & $50.6 \%$ & $0.0 \%$ & ND & $0.0 \%$ & $0.0 \%$ \\
\hline $10: 90(10 \%)$ & $9.8 \%$ & $5.0 \%$ & $87.7 \%$ & $96.6 \%$ & $45.1 \%$ & $91.5 \%$ \\
\hline $5: 95(5 \%)$ & $4.5 \%$ & ND & $94.0 \%$ & $97.7 \%$ & $46.0 \%$ & $95.5 \%$ \\
\hline $1: 99(1 \%)$ & ND & ND & $97.5 \%$ & $99.0 \%$ & $49.9 \%$ & $98.1 \%$ \\
\hline $0.1: 99.9(0.1 \%)$ & ND & ND & $97.8 \%$ & $100.0 \%$ & $52.5 \%$ & $98.9 \%$ \\
\hline call type & novel & novel & targeted & novel & targeted & targeted \\
\hline percent in undiluted BxPC-3 & ND & ND & $98.0 \%$ & $100.0 \%$ & $48.2 \%$ & $100.0 \%$ \\
\hline \multicolumn{2}{|c|}{} & \multicolumn{1}{|c|}{} & & & \\
\hline HPAF-II:BxPC-3 (\%HPAF) & KRAS & PIK3CA & RET (1) & RET (2) & TP53 (1) & TP53 (2) \\
\hline Undiluted (100\%) & $75.9 \%$ & $100.0 \%$ & $100.0 \%$ & $100.0 \%$ & $0.0 \%$ & $98.1 \%$ \\
\hline $10: 90(10 \%)$ & $15.4 \%$ & $15.9 \%$ & $8.7 \%$ & $9.1 \%$ & $90.8 \%$ & $8.5 \%$ \\
\hline $5: 95(5 \%)$ & $8.3 \%$ & $9.2 \%$ & $5.7 \%$ & $5.6 \%$ & $95.1 \%$ & $5.1 \%$ \\
\hline $1: 99(1 \%)$ & $0.0 \%$ & ND & ND & ND & $98.8 \%$ & ND \\
\hline $0.1: 99.9(0.1 \%)$ & $0.0 \%$ & ND & ND & ND & $100.0 \%$ & ND \\
\hline call type & targeted & novel & novel & novel & targeted & novel \\
\hline percent in undiluted BxPC-3 & $0.0 \%$ & ND & ND & ND & $100.0 \%$ & ND \\
\hline
\end{tabular}

ND - not detected 
Table 5. Percentage of reads in each gene that were flagged NO CALL.

\begin{tabular}{|c|c|}
\hline Gene & $\begin{array}{c}\text { Percentage } \\
\text { no call }\end{array}$ \\
\hline ABL1 & $0.0 \%$ \\
\hline AKT1 & $0.0 \%$ \\
\hline ALK & $0.0 \%$ \\
\hline APC & $2.5 \%$ \\
\hline ATM & $0.0 \%$ \\
\hline BRAF & $0.0 \%$ \\
\hline $\mathrm{CDH} 1$ & $14.3 \%$ \\
\hline CDKN2A & $0.0 \%$ \\
\hline CSF1R & $0.0 \%$ \\
\hline CTNNB1 & $0.9 \%$ \\
\hline EGFR & $0.0 \%$ \\
\hline ERBB2 & $0.0 \%$ \\
\hline ERBB4 & $0.0 \%$ \\
\hline $\mathrm{EZH} 2$ & $0.0 \%$ \\
\hline FBXW7 & $2.0 \%$ \\
\hline FGFR1 & $0.0 \%$ \\
\hline FGFR2 & $0.0 \%$ \\
\hline FGFR3 & $0.0 \%$ \\
\hline FLT3 & $1.3 \%$ \\
\hline GNA11 & $0.0 \%$ \\
\hline GNAS & $2.1 \%$ \\
\hline GNAQ & $0.0 \%$ \\
\hline HNF1A & $0.0 \%$ \\
\hline HRAS & $0.0 \%$ \\
\hline IDH1 & $0.0 \%$ \\
\hline IDH2 & $0.0 \%$ \\
\hline JAK2 & $0.0 \%$ \\
\hline JAK3 & $0.0 \%$ \\
\hline KDR & $0.0 \%$ \\
\hline KIT & $0.2 \%$ \\
\hline KRAS & $2.9 \%$ \\
\hline MET & $0.0 \%$ \\
\hline MLH1 & $0.0 \%$ \\
\hline MPL & $12.5 \%$ \\
\hline NOTCH1 & $5.0 \%$ \\
\hline NPM1 & $0.0 \%$ \\
\hline NRAS & $6.8 \%$ \\
\hline PDGFRA & $9.1 \%$ \\
\hline PIK3CA & $0.1 \%$ \\
\hline PTEN & $3.4 \%$ \\
\hline PTPN11 & $0.0 \%$ \\
\hline RB1 & $0.0 \%$ \\
\hline RET & $0.0 \%$ \\
\hline SMAD4 & $0.0 \%$ \\
\hline SMARCB1 & $0.0 \%$ \\
\hline SMO & $0.0 \%$ \\
\hline SRC & $0.0 \%$ \\
\hline STK11 & $5.4 \%$ \\
\hline TP53 & $1.7 \%$ \\
\hline VHL & $2.7 \%$ \\
\hline
\end{tabular}




\section{References}

Bolotin, D. A., I. Z. Mamedov, O. V. Britanova, I. V. Zvyagin, D. Shagin, S. V. Ustyugova, M. A. Turchaninova, S. Lukyanov, Y. B. Lebedev and D. M. Chudakov (2012). "Next generation sequencing for TCR repertoire profiling: platformspecific features and correction algorithms." Eur. J Immunol 42(11): 3073-3083 DOI: 10.1002/eji.201242517.

Bragg, L. M., G. Stone, M. K. Butler, P. Hugenholtz and G. W. Tyson (2013). "Shining a light on dark sequencing: characterising errors in Ion Torrent PGM data." PLoS Comput Biol 9(4): e1003031 DOI: 10.1371/journal.pcbi.1003031.

Deer, E. L., J. Gonzalez-Hernandez, J. D. Coursen, J. E. Shea, J. Ngatia, C. L. Scaife, M. A. Firpo and S. J. Mulvihill (2010). "Phenotype and Genotype of Pancreatic Cancer Cell Lines." Pancreas 39: 425-435 DOI: 10.1097/MPA.0b013e3181c15963.

Fassan, M., M. Simbolo, E. Bria, A. Mafficini, S. Pilotto, P. Capelli, M. Bencivenga, S. Pecori, C. Luchini, D. Neves, G. Turri, C. Vicentini, L. Montagna, A. Tomezzoli, G. Tortora, M. Chilosi, G. De Manzoni and A. Scarpa (2014). "Highthroughput mutation profiling identifies novel molecular dysregulation in high-grade intraepithelial neoplasia and early gastric cancer." Gastric Cancer 17: 442-449 DOI: 10.1007/s10120-013-0315-1.

Geurts-Giele, W. R., A. W. Dirkx-van der Velden, N. M. Bartalits, L. C. Verhoog, W. E. Hanselaar and W. N. Dinjens (2013). "Molecular diagnostics of a single multifocal non-small cell lung cancer case using targeted next generation sequencing." Virchows Arch. 462(2): 249-254 DOI: 10.1007/s00428-012-1346-4.

Hadd, A. G., J. Houghton, A. Choudhary, S. Sah, L. Chen, A. C. Marko, T. Sanford, K. Buddavarapu, J. Krosting, L. Garmire, D. Wylie, R. Shinde, S. Beaudenon, E. K. Alexander, E. Mambo, A. T. Adai and G. J. Latham (2013). "Targeted, high-depth, next-generation sequencing of cancer genes in formalin-fixed, paraffin-embedded and fine-needle aspiration tumor specimens." J Mol. Diagn. 15(2): 234-247 DOI: 10.1016/j.jmoldx.2012.11.006.

Harrington, C. T., E. I. Lin, M. T. Olson and J. R. Eshleman (2013). "Fundamentals of pyrosequencing." Arch. pathol. lab. med. 137(9): 1296-1303 DOI: 10.5858/arpa.2012-0463-RA.

Kim, H. S., J. S. Sung, S. J. Yang, N. J. Kwon, L. Jin, S. T. Kim, K. H. Park, S. W. Shin, H. K. Kim, J. H. Kang, J. O. Kim, J. Y. Park, J. E. Choi, H. Yoon, C. K. Park, K. S. Yang, J. S. Seo and Y. H. Kim (2013). "Predictive Efficacy of Low Burden EGFR Mutation Detected by Next-Generation Sequencing on Response to EGFR Tyrosine Kinase Inhibitors in NonSmall-Cell Lung Carcinoma." PLoS One 8(12): e81975 DOI: 10.1371/journal.pone.0081975.

Lee, C. H., L. N. Hoang, S. Yip, C. Reyes, A. Marino-Enriquez, G. Eilers, D. Tao, S. Chiang, J. A. Fletcher, R. A. Soslow, M. R. Nucci and E. Oliva (2014). "Frequent expression of KIT in endometrial stromal sarcoma with YWHAE genetic rearrangement." Mod. Pathol. 27: 751-757 DOI: 10.1038/modpathol.2013.199.

Loman, N. J., R. V. Misra, T. J. Dallman, C. Constantinidou, S. E. Gharbia, J. Wain and M. J. Pallen (2012). "Performance comparison of benchtop high-throughput sequencing platforms." Nat. Biotech. 30(5): 343-349 DOI: 10.1038/nbt.2198.

Luthra, R., K. P. Patel, N. G. Reddy, V. Haghshenas, M. J. Routbort, M. A. Harmon, B. A. Barkoh, R. Kanagal-Shamanna, F. Ravandi, J. E. Cortes, H. M. Kantarjian, L. J. Medeiros and R. R. Singh (2014). "Next generation sequencing based multi-gene mutational screen for acute myeloid leukemia using miseq: applicability for diagnostics and disease monitoring." Haematol. 99: 465-473 DOI: 10.3324/haematol.2013.093765.

Malapelle, U., E. Vigliar, R. Sgariglia, C. Bellevicine, L. Colarossi, D. Vitale, P. Pallante and G. Troncone (2014). "Ion Torrent next-generation sequencing for routine identification of clinically relevant mutations in colorectal cancer patients." J. Clin. Pathol. 68(1): 64-68 DOI: 10.1136/jclinpath-2014-202691.

Nguyen-Dumont, T., Z. L. Teo, B. J. Pope, F. Hammet, M. Mahmoodi, H. Tsimiklis, N. Sabbaghian, M. Tischkowitz, W. D. Foulkes, K. C. F. C. f. r. i. F. B. c. (kConFab), G. G. Giles, J. L. Hopper, A. B. C. F. Registry, M. C. Southey and D. J. Park (2013). "Hi-Plex for high-throughput mutation screening: application to the breast cancer susceptibility gene PALB2." BMC Med. Genomics 6(48) DOI: 10.1186/1755-8794-6-48.

Parson, W., C. Strobl, G. Huber, B. Zimmermann, S. M. Gomes, L. Souto, L. Fendt, R. Delport, R. Langit, S. Wootton, R. Lagacé and J. Irwin (2013). "Evaluation of next generation mtGenome sequencing using the lon Torrent Personal Genome Machine (PGM)." Forensic Sci. Int. Genet. 7(5): 543-549 DOI: 10.1016/j.fsigen.2013.06.003.

Quail, M. A., M. Smith, P. Coupland, T. D. Otto, S. R. Harris, T. R. Connor, A. Bertoni, H. P. Swerdlow and Y. Gu (2012). "A tale of three next generation sequencing platforms: comparison of Ion Torrent, Pacific Biosciences and Illumina MiSeq sequencers." BCM Genomics 13: 341 DOI: 10.1186/1471-2164-13-341. 
Robinson, J. T., H. Thorvaldsdóttir, W. Winckler, M. Guttman, E. S. Lander, G. Getz and J. P. Mesirov (2011). "Integrative Genomics Viewer." Nat. Biotech. 29(24-26) DOI: 10.1038/nbt.1754.

Scarpa, A., K. Sikora, M. Fassan, A. M. Rachiglio, R. Cappellesso, D. Antonello, E. Amato, A. Mafficini, M. Lambiase, C. Esposito, E. Bria, F. Simonato, M. Scardoni, G. Turri, M. Chilosi, G. Tortora, A. Fassina and N. Normanno (2013). "Molecular typing of lung adenocarcinoma on cytological samples using a multigene next generation sequencing panel." PLoS One 8(11): e80478 DOI: 10.1371/journal.pone.0080478.

Seo, S. B., J. L. King, D. H. Warshauer, C. P. Davis, J. Ge and B. Budowle (2013). "Single nucleotide polymorphism typing with massively parallel sequencing for human identification." Int. J. Leg. Med. 127(6): 1079-1086 DOI: 10.1007/s00414-013-0879-7.

Singh, R. R., K. P. Patel, M. J. Routbort, N. G. Reddy, B. A. Barkoh, B. Handal, R. Kanagal-Shamanna, W. O. Greaves, L. J. Medeiros, K. D. Aldape and R. Luthra (2013). "Clinical validation of a next-generation sequencing screen for mutational hotspots in 46 cancer-related genes." J. Mol. Diagn. 15(5): 607-622 DOI: 10.1016/j.jmoldx.2013.05.003.

Song, S., K. Nones, D. Miller, I. Harliwong, K. S. Kassahn, M. Pinese, M. Pajic, A. J. Gill, A. L. Johns, M. Anderson, O. Holmes, C. Leonard, D. Taylor, S. Wood, Q. Xu, F. Newell, M. J. Cowley, J. Wu, P. Wilson, L. Fink, A. V. Biankin, N. Waddell, S. M. Grimmond and J. V. Pearson (2012). "qpure: A tool to estimate tumor cellularity from genomewide single-nucleotide polymorphism profiles." PLoS One 7(9): e45835 DOI: 10.1371/journal.pone.0045835.

Tarabeux, J., B. Zeitouni, V. Moncoutier, H. Tenreiro, K. Abidallah, S. Lair, P. Legoix-Né, Q. Leroy, E. Rouleau, L. Golmard, E. Barillot, M. H. Stern, T. Rio-Frio, D. Stoppa-Lyonnet and C. Houdayer (2014). "Streamlined ion torrent PGMbased diagnostics: BRCA1 and BRCA2 genes as a model." Eur. J Hum. Genet. 22: 535-541 DOI: 10.1038/ejhg.2013.181.

Tenedini, E., I. Bernardis, V. Artusi, L. Artuso, E. Roncaglia, P. Guglielmelli, L. Pieri, C. Bogani, F. Biamonte, G. Rotunno, C. Mannarelli, E. Bianchi, A. Pancrazzi, T. Fanelli, G. Malagoli Tagliazucchi, S. Ferrari, R. Manfredini, A. M. Vannucchi and E. Tagliafico (2014). "Targeted cancer exome sequencing reveals recurrent mutations in myeloproliferative neoplasms." Leuk. 28: 1052-1059 DOI: 10.1038/leu.2013.302.

Thorvaldsdóttir, H., J. T. Robinson and J. P. Mesirov (2012). "Integrative Genomics Viewer (IGV): high-performance genomics data visualization and exploration. ." Brief. Bioinform. 14: 178-192 DOI: 10.1093/bib/bbs017.

Yousem, S. A., S. Dacic, Y. E. Nikiforov and M. Nikiforova (2013). "Pulmonary Langerhans Cell Histiocytosis: Profiling of Multifocal Tumors Using Next-Generation Sequencing Identifies Concordant Occurrence of BRAF V600E Mutations." Chest 143(6): 1679-1684 DOI: 10.1378/chest.12-1917.

Reference word count: 1172 